\title{
Combined inhibition of Bruton's Tyrosine Kinase (BTK) and Phosphoinositide 3-kinase (PI3K) p110ס improves monocytosis and splenomegaly in a JMML Mouse Model
}

\author{
Roshni Patel ${ }^{1}$, Baskar Ramdas², Lisa Deng ${ }^{1}$, Victoria Jideonwo-Auman², Reuben \\ Kapur $^{2}$ \\ ${ }^{1}$ Indiana University School of Medicine, ${ }^{2}$ Herman B Wells Center for Pediatric \\ Research, Indiana University School of Medicine
}

Background and Hypothesis: Juvenile myelomonocytic leukemia (JMML) is an aggressive, childhood leukemic disorder for which there are no efficacious chemotherapeutics. Gain-of-function (GOF) mutations in the SHP2 phosphatase oncogene, Ptpn11, are the most common associated mutations. In hematopoietic cells, these mutations lead to increased AKT and ERK activation, which results in hyperproliferation of myeloid cells. Clinically, this manifests as monocytic leukocytosis and marked splenomegaly with the consequence of severe thrombocytopenia. Previously, this lab has shown that pharmacological inhibition of p110d, the hematopoietic-specific catalytic subunit of PI3K, moderates monocytosis and splenomegaly in a JMML mouse model with a SHP2 GOF mutation (E76K mice). Additionally, BTK has been identified as a potential therapeutic target as it cooperates with PI3K to increase activation of AKT and ERK in myeloid cells. Using a dual-hit approach of targeting both PI3K p110d and BTK may serve as a valuable treatment design for JMML.

Experimental Design or Project Methods: We have treated E76K mice with a combination of $20 \mathrm{mg} / \mathrm{kg}$ ACP319, a PI3K p110d-specific inhibitor, and $20 \mathrm{mg} / \mathrm{kg}$ acalabrutinib, a BTK-specific inhibitor, via oral gavage twice daily for 21 days and performed hematopoietic analysis including the degree of splenomegaly, monocytosis, and thrombocytopenia.

Results: The combination treatment scheme significantly decreased monocytosis and ameliorated thrombocytopenia compared to vehicle treated mice. Furthermore, splenomegaly was significantly reduced in the combinationtreated mice compared to vehicle.

Conclusion and Potential Impact: Combination chemotherapy with PI3K p110d-and BTK-specific inhibitors profoundly corrects disease state hallmarks of JMML, and may warrant further clinical investigation of efficacy. 